\title{
What Can We Learn from the Shapes of Secondary Electron Puddles on Direct Electron Detectors?
}

\author{
Abhik Datta ${ }^{1}$, See Wee Chee ${ }^{1,2,3}$, Benjamin Bammes ${ }^{4}$, Liang Jin ${ }^{4}$ and Duane Loh ${ }^{1,2}$ \\ 1. Centre for BioImaging Sciences and Department of Biological Sciences, National University of \\ Singapore, Singapore 117557 \\ 2. Department of Physics, National University of Singapore, Singapore 117551 \\ 3. Centre for Advanced 2D Materials and Graphene Research Centre, National University of Singapore, \\ Singapore 117546 \\ 4. Direct Electron, LP, San Diego, CA, USA 92128
}

Direct electron detectors are a recent breakthrough in transmission electron microscopy that have opened up the possibility of super-resolution microscopy even at low doses. These detectors use CMOS sensors that allow fast read-outs, such that individual electron events (the "puddles" formed by the secondary electrons) can be recorded.

While single event detection based imaging, referred to as electron counting, is starting to be widely adopted, several challenges still remain. One of these is, accurately identifying the entry point of the primary electron given the secondary electron puddle produced by it. Succeeding here reduces imaging noise, improves detector efficiency and allows for super-pixel accurate reconstruction [1]. It has been shown that these secondary electron puddles can vary greatly in shape and size [2]. While several techniques have been proposed [2], there is no consensus on which of these is optimal and why they would be better. Here, we investigate the shape and size of these secondary electron puddles to better understand how electron counting can be improved.

We present results from several shadow experiments, where a beam blanker is used to block a part of the electron beam, performed using the DE-16 detector (Direct Electron, LP, San Diego, CA, USA) on a JEM2200FS transmission electron microscope (JEOL USA Inc, Peabody, MA, USA). These experiments are used to study the differences between the secondary electron puddles in the exposed region and the shadow of the beam blanker (Fig. 1). Indeed in our experiments significant number of secondary electron puddles could be seen in the shadow region, which was previously conjectured to arise from electrons scattered back into the detector from the detector's surface, electrons scattered by the microscope column, and Xray photons generated when electrons are absorbed by the beam blanker and the microscope column [3].

We found a statistically significant difference between the distribution of sizes of secondary electron puddles in the exposed and shadow regions (Fig. 2a). The puddles in the shadow region have larger variance in size than puddles in the exposed region. Although the secondary electron puddles vary greatly in shape, they appear to share common morphological features (Fig. 2c,d). Further, there appears no statistically significant differences in the shape of electron puddles found in the exposed versus shadow regions. We quantify the frequency of such stray electrons for the DE-16 detector (Fig. 2b), and speculate on possible strategies for noise reduction.

References:

[1]. G. McMullan, A.R. Faruqi and D. Clare, et al., Ultramicroscopy 147, 156-163 (2014)

[2]. G. McMullana, A.T. Clark, R. Turchetta, et al., Ultramicroscopy 109, 1411-1416 (2009) 
[3]. L. Jin, UC San Diego Electronic Theses and Dissertations (2009) b6636471

[4]. The authors would like to acknowledge the use of the EM facility at the NUS Center for BioImaging Sciences, and help from Ping Lee Chong and Jian Shi.

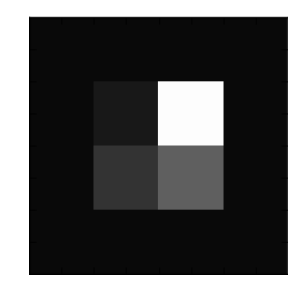

Sum: 2052 Ma

(a)

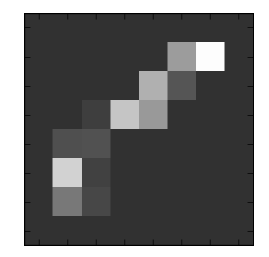

Sum: 1120 Max: 255

(c)

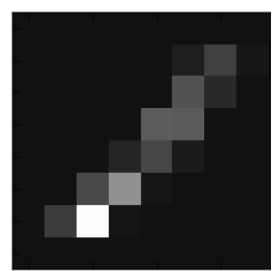

(b)

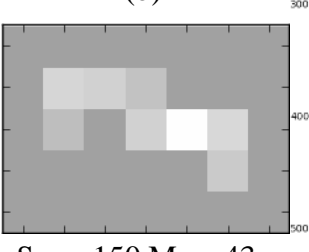

(d)

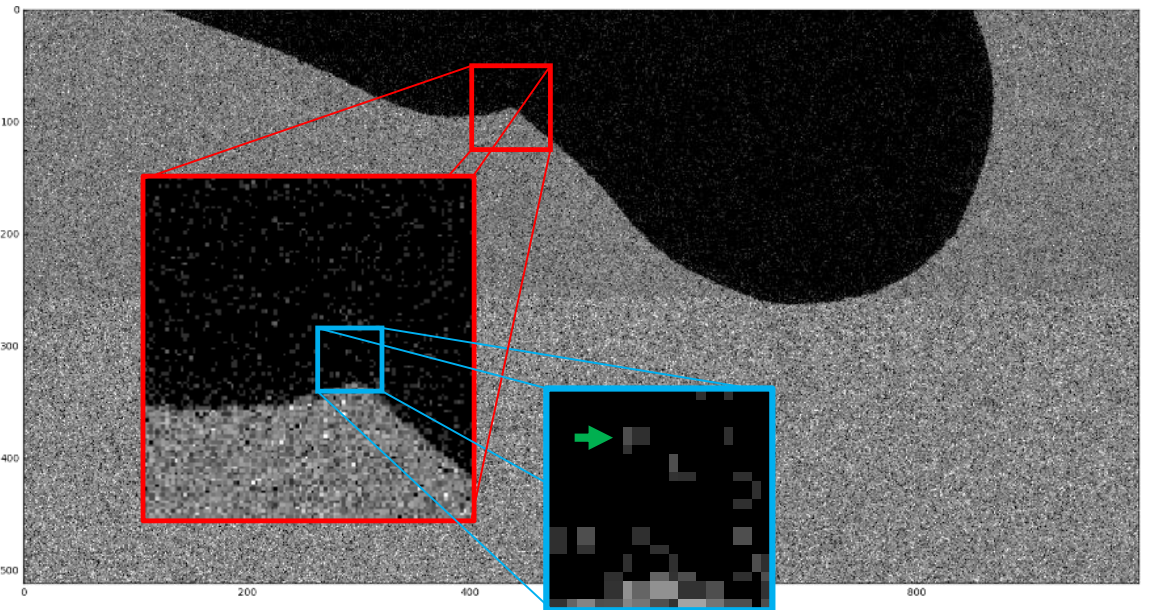

(e)

Figure 1. Secondary electron puddles and counted image. (a)-(d) Secondary electron puddles. The images are scaled such that their maximum pixel intensities have an ADU of 255. The maximum and total ADUs are listed below the images (e) A $500 \times 1000$ pixel area of a counted image obtained by summing the electron counting outputs of 48,324 frames. The beam blanker's shadow is visible in the top half of the image. The green arrow in the bottom inset points to a secondary electron puddles in the shadow region.

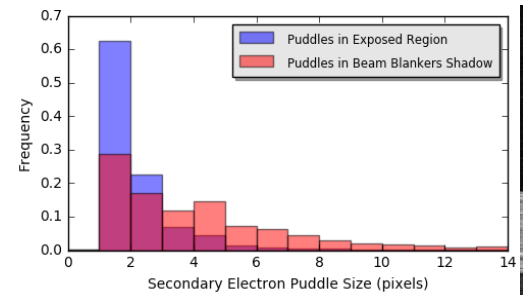

(a)

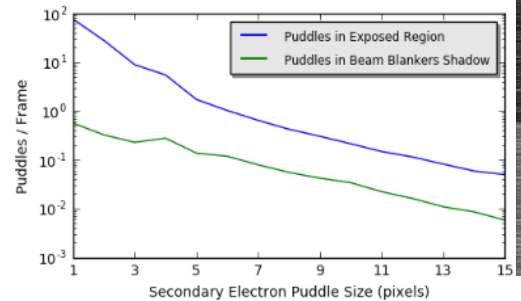

(b)

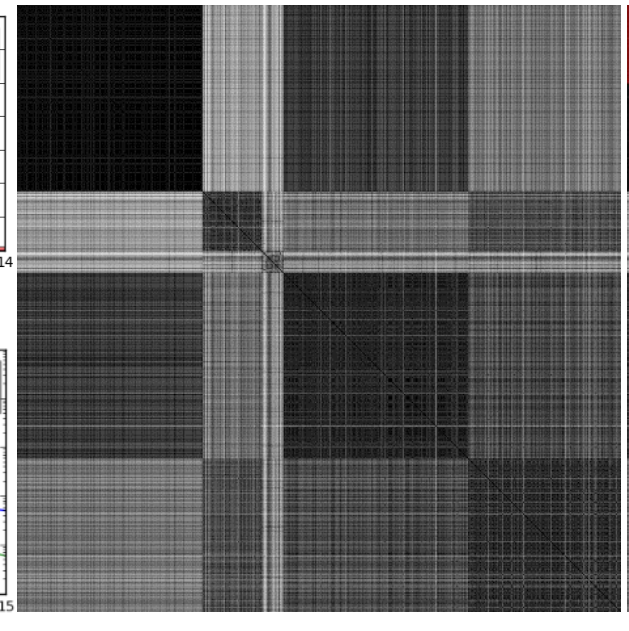

(c)

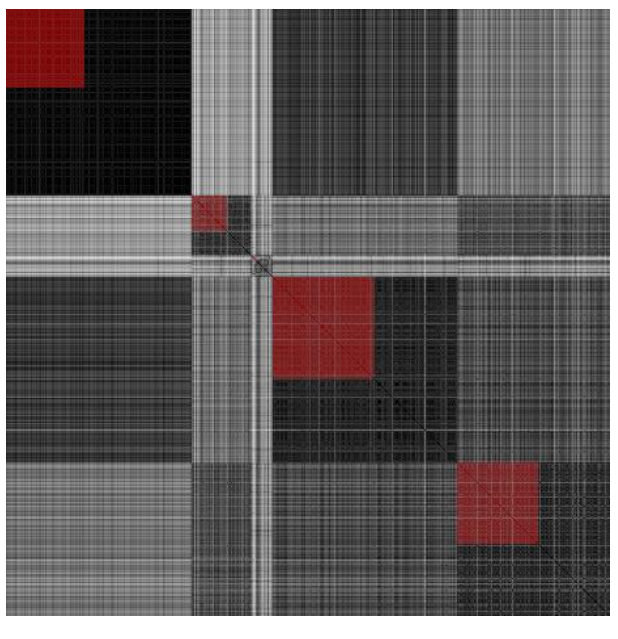

(d)

Figure 2. Shape and frequency analyses of secondary electron puddle sizes and shapes. (a) The distribution of size of 20,000 secondary electron puddles extracted from the exposed and shadow regions. (b) Average frequency of secondary electron puddle sizes in each frame. (c) Euclidean distance matrix $M$ whose $i j^{\text {th }}$ element is the dissimilarity between the shapes of the $i^{\text {th }}$ and $j^{\text {th }}$ secondary electron puddles of area 5 pixels. The distances were computed using pixel intensities as features. The puddles were rotated so that their principal axes are aligned with the $x$-axis before computing the distances. Equal numbers of puddles were randomly chosen from shadow and exposed regions. The rows and columns of $M$ are sorted such that secondary electron puddles that belong to the same cluster appear together. The darker diagonal blocks represent puddles that are morphologically similar. (d) Same as (c) except the puddles from the shadow region are highlighted in red. 